\title{
GPS normalization and preliminary modeling results of total electron content during a midlatitude space weather event
}

\author{
Jonathan J. Makela and Michael C. Kelley \\ School of Electrical and Computer Engineering, Cornell University, Ithaca, New York
}

Jan J. Sojka

Space Environment Corporation, Logan, Utah

Xiaoqing Pi and Anthony J. Mannucci

Jet Propulsion Laboratory, Pasadena, California

\begin{abstract}
On November 22-23, 1997, a geomagnetic storm occurred during a period of excellent viewing conditions over the Arecibo Observatory in Puerto Rico. Here we explore the total electron content (TEC) registered by Global Positioning System (GPS) receivers located close to the Cornell All-Sky Imager (CASI) at the Arecibo Observatory. The storm began with the equatorward surge of a very high (100\% increase) TEC enhancement stretching for many hours of local time on the dayside. At dusk the TEC over the Caribbean remained elevated with levels equal to the noontime monthly averages. During the event the TEC was highly structured and clearly correlated with high and low airglow emission levels. In one fortuitous instance a common ionospheric penetration point (15 km apart), shared by two GPS satellites viewed from two receiving stations, registered an 8 TEC unit difference during the active period. We show that a GPS station can be calibrated using the pseudorange method and a reliable data-driven technique during quiet conditions and still have absolute TEC capability within 2 TEC units (RMS) 5 days later. We compare the observations to a climatological model which, although reasonable for quiet times, is very poor during the storm period. We also present an independent evaluation of the GPS TEC. This study is an initial step toward quality control of this database, needed before it is used in an assimilation model.
\end{abstract}

\section{Introduction}

One goal of the space science community is to build data assimilative models for the ionosphere similar to those used to forecast meteorological conditions. A key element for such models is almost certainly going to be data from the Global Positioning System (GPS). With 27 operational satellites and hundreds

Copyright 2001 by the American Geophysical Union.

Paper number 1999RS002427.

0048-6604/01/1999RS002427\$11.00 of ground sites, a considerable database consisting of total electron content (TEC) data can be obtained. This line-of-sight TEC, among other parameters, can then be ingested into a data assimilative model.

In this paper we explore one method of extracting such data and apply it to a very interesting period in November 1997. We study a 5 day period overlapping with the November 1997 Caribbean Combined Ionospheric Campaigns (CIC), during which a magnetic storm occurred. This storm doubled the daytime TEC over a vast region in the Atlantic sector and resulted in nighttime TEC values comparable to quiet noontime values. We also present a preliminary 
modeling effort, basically showing how present models deal poorly with geomagnetic disturbance effects at midlatitudes. Two other studies of this event have already been published [Kelley et al., 2000; Garcia et al., 2000], which discussed these large-scale TEC disturbances as well as the mesoscale structure over Puerto Rico as revealed by an all-sky camera using a $630 \mathrm{~nm}$ filter.

\section{GPS Technique Description and Validation}

Since our primary goal is to study the spatial and temporal variability of the TEC, we sought to develop a simple technique that would permit absolute TEC determination from several sites with high time resolution. We used the pseudorange technique as discussed by Lanyi and Roth [1988] to accomplish this goal, which was then enhanced by using the global ionospheric mapping (GIM) technique developed at the Jet Propulsion Laboratory (JPL). The GIM technique was used to remove the biases inherent in GPS TEC calculations. Once the biases were determined during a quiet period for a given satellitereceiver pair, we hypothesized that they would be valid over the subsequent storm period. Such a method has considerable potential for space weather applications related to GPS model performance during severe conditions as well as for other applications requiring highly resolved temporal and spatial TEC data. For the spatial studies we concentrate on two receivers in the Caribbean. The first is on the island of St. Croix, Virgin Islands, operated by JPL and part of the International GPS Service (IGS). The second receiver is at the Coast Guard station in Isabela, Puerto Rico (roughly $250 \mathrm{~km}$ west of the St. Croix receiver), and is part of the Continuously Operating Reference Station (CORS) network. Both the IGS and CORS networks make data available on the World Wide Web.

The Global Positioning System, when used with dual-frequency receivers, has been known for quite some time to make very accurate relative TEC measurements. There is, however, quite a bit of controversy over the best way to extract absolute values. In this paper, we have chosen what we will call the pseudorange technique [Lanyi and Roth, 1988], which is summarized next.

Because of the dispersive nature of the ionosphere the two $L$ band radio signals transmitted by each GPS satellite ( $L 1$ at $1.5754 \mathrm{GHz}$ and $L 2$ at 1.2276
$\mathrm{GHz}$ ) are delayed by different amounts as they propagate from the satellite to the receiver. These delays show up as a difference in the pseudorange and phase information, both of which are determined by a dual-frequency receiver. The pseudorange information obtained from the signal delay has the advantage of giving an absolute TEC value. However, these values are strongly influenced by multipath and system noise, resulting in extremely noisy TEC calculations. Using phase information obtained from the signal phase advance yields a much cleaner TEC measurement but loses the absolute value because of integer cycle ambiguities in phase, which are fundamental to oscillators, including the ones used in GPS satellites and receivers. By setting the mean of the TEC calculated with phase information to the mean of the TEC calculated with pseudorange information, we can combine the accuracy of the phase measurements with the absolute values of the pseudorange measurements. To minimize the effects of multipath in the pseudorange measurements (which may have nonzero mean), we do not use any part of the satellite pass that falls below $25^{\circ}$ elevation in the offset determination.

This procedure leaves two errors in our TEC estimation, both of which are due to the hardware. These errors are caused by a differential delay between the two GPS frequencies in both the receiver and satellite hardware. The resulting biases must be removed if absolute TEC measurements are to be made accurately. We can model the raw, line-of-sight TEC as

$$
\mathrm{TEC}_{p}=\mathrm{TEC}_{\mathrm{los}}+b_{r}+b_{\imath}
$$

where $\mathrm{TEC}_{p}$ is the TEC obtained using the pseudorange technique detailed above; $\mathrm{TEC}_{\mathrm{los}}$ is the actual, unbiased line-of-sight TEC; $b_{r}$ is the receiver bias, and $b_{i}$ is the satellite bias (each of the 27 GPS satellites has its own bias). Both the TEC and biases are measured in TEC units (1 TECU $\left.=10^{16} \mathrm{e} / \mathrm{m}^{2}\right)$.

The global ionospheric mapping (GIM) technique developed at JPL [e.g., Wilson et al., 1995; Ho et al., 1996; Mannucci et al., 1998] provides a way to remove both the receiver and satellite biases. Using an array of receivers spread across the globe, a map of the absolute vertical TEC is created. In the process of creating this map the biases $\left(b_{r}\right.$ and $\left.b_{i}\right)$ are solved for and removed. Line-of-sight data corrected in this way were provided to us for the period of November 20-25, 1997, for the St. Croix site. If we take the difference between TEC obtained from the GIM 
technique, which we assume to be $\mathrm{TEC}_{\text {los }}$, and that obtained from the pseudorange technique for each satellite-receiver pair at St. Croix, we find the combined satellite and receiver bias for St. Croix:

$$
\mathrm{TEC}_{p, s c}-\mathrm{TEC}_{\mathrm{GIM}}=b_{r, s c}+b_{i},
$$

where the subscript $s c$ refers to the St. Croix site.

Next, we need to find the combined satellite and receiver bias for the Isabela, Puerto Rico site, which was not included in the GIM technique at this time. If we assume that the line-of-sight TEC for a given satellite is the same for the two sites (separated by $250 \mathrm{~km}$ ), we can take the difference between the pseudorange-technique-derived TEC for each receiver for a given satellite to remove both $\mathrm{TEC}_{\mathrm{los}}$ and $b_{i}$ :

$$
\mathrm{TEC}_{p, p r}-\mathrm{TEC}_{p, s c}=b_{r, p r}-b_{r, s c}
$$

where the subscript $p r$ refers to the Isabela site. The assumption that the line-of-sight TEC is the same at both stations should be valid for quiet time conditions, especially at night when the ionosphere is fairly uniform and well behaved. This difference is taken for each satellite seen by both stations. Ideally, this difference should be the same for each satellite, as it is only dependent on the receiver hardware. Consequently, the resulting nighttime differences (one for each satellite) are averaged to give our "best guess" difference between receiver biases. For this data set we find this difference to be -7.5 TECU.

The final step in finding the combined satellite and receiver biases for the receiver at Isabela, Puerto Rico, is to add the St. Croix satellite and receiver biases (equation (2)) to the difference between the two receiver biases (equation (3)):

$$
\left(b_{r, s c}+b_{\imath}\right)+\left(b_{r, p r}-b_{r, s c}\right)=b_{r, p r}+b_{i}
$$

This is what must be subtracted from the pseudorange technique-derived TEC for Isabela, described by (1), to obtain the absolute, unbiased line-of-sight TEC for this receiver. Obtained in this way for both sites on a quiet calibration day, the combined satellite and receiver biases (equations (2) and (4)) were then used for the remaining days in the study to calculate absolute unbiased TEC.

We chose November 20 for the calibration day. The GIM technique was run on this period at JPL. Satellite 19 as viewed from St. Croix and satellite 15 as viewed from Isabela are of particular interest: for the 6 consecutive days the ionospheric penetration points
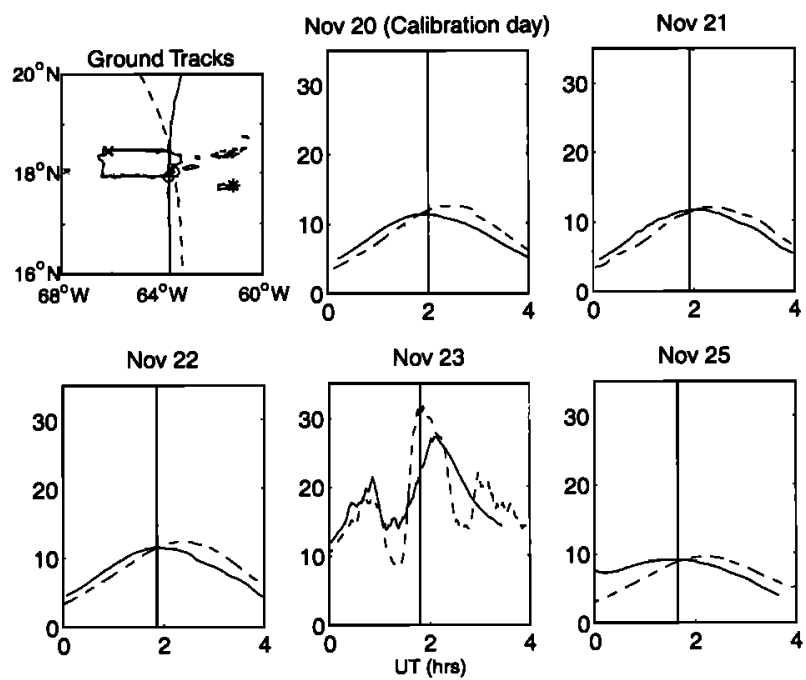

Figure 1. Vertical TEC for satellite 19 as viewed from St. Croix (dashed curves; moving north to south) and satellite 15 as viewed from Isabela (solid curves; moving south to north). The top left panel shows the ground traces of each satellite's ionospheric pierce point ( 350 $\mathrm{km})$. Note that the traces continue to both the north and the south of the region shown here. The circle on each trace is the location of each satellite at the time of closest passing, corresponding to the vertical lines on the other five plots. The cross is the location of the receiver at Isabela, Puerto Rico. The asterisk is the location of the receiver on St. Croix. The time period plotted is 0000-0400 UT (2000-0000 LT). November 24 is missing because the Isabela receiver was down during the time period of interest.

at $350 \mathrm{~km}$ came quite close to each other (within approximately $15 \mathrm{~km}$ ). This can be seen in the top left panel of Figure 1, where the $350 \mathrm{~km}$ penetration points of both satellites are plotted as a function of time for November 20. Similar plots for the other days of this period are essentially indistinguishable from this one. When interpreting the plots, it is important to note that the two satellites are moving in opposite directions.

The rest of the panels in Figure 1 display absolute vertical TEC under the moving penetration point, using a standard algorithm for converting line-ofsight to vertical TEC [Sardon and Zarraoa, 1997]. For the moment, we concentrate on the quiet days, leaving November 23 for the next paragraph. Nearperfect agreement between the two data sets was found at the common pierce point on November 22 . Not only were the two absolute TEC values well within 1 unit at the time of the vertical line, but 
also there is near-perfect symmetry about the crossing point. This indicates that over the 4 hour period and $8^{\circ}$ of latitude covered, the latitudinal gradient was nearly constant and there was very little local time dependence in the TEC. Both traces suggest that the ionosphere has a larger TEC south of Puerto Rico. This may be explained by the satellite lines of sight tracing a latitudinal gradient or entering or leaving the equatorial anomaly zone.

On November 21 the two curves cross later in time than on November 22. At the time of TEC equality, satellite 15 (viewed from the east) was north of the closest penetration point while satellite 19 (viewed from the west) was south of that point. Likewise, there is a larger difference between the two curves an hour before the TEC crossing point than an hour afterward. Both observations suggest a less uniform ionosphere on November 21 and a similar conclusion holds for November 20.

November 25 has a pronounced north-south gradient and a larger local time dependence than the days before the storm event. November 28 has no Isabela data, so it is excluded from this discussion. We conclude that small TEC differences at the times corresponding to the vertical line simply correspond to small changes of the TEC when viewed from different locations because of either temporal or spatial variations. An incorrect choice of penetration altitude could also contribute to the small differences.

November 23 is remarkable for a variety of reasons. Notice in particular that the absolute TEC is much higher than during the other days and is extremely variable. In fact, the variations are larger than the absolute TEC on the other days. Clearly, a major space weather event is occurring. At the time of the vertical line the absolute TEC differed by 8 units when at most it should have been 1 or 2 units, on the basis of the other days presented here. Huge variations in TEC thus occur on midlatitude paths with the same ionospheric penetration point but different receiver viewpoints separated by only a few hundred kilometers. Conversely, as discussed in more detail below, different satellites viewed from the same location will be affected by quite different TEC along the line-of-sight paths, even when corrected for slant differences, as done in Figure 1. It is interesting to note that the average absolute TEC between 0000 and $0030 \mathrm{UT}$ for satellite 15 is very nearly the same as that for satellite 19 around 0300-0400 UT. In both cases the lines-of-sight are well to the south of Puerto Rico. These regions are structured at the level of 3-5
TEC units (peak to peak) but do not have the huge variations seen nearer to the center of the plots.

We wish to further emphasize the robust nature of the receiver-satellite pairs when normalized on a quiet day. Even after 5 days, the absolute TEC for these two different satellites, viewed from two different receivers, is in clear and remarkable agreement.

To explore this result in more detail, we look at a subset of six satellites seen from the St. Croix site. Again, we use November 20 as our calibration day to obtain the biases for each satellite. We keep these biases unchanged for both the storm night (November 22-23) and a calm night following the storm (November 24-25). As stated before, our hypothesis is that the biases obtained on the previous calm day should remain the same for subsequent days. Our results on the calm day at the end of the period should be in reasonable agreement with an independent application of the GIM technique on that day. By looking at the storm night we will be able to evaluate how the GIM technique handles periods with extreme TEC fluctuations and ionospheric gradients.

Table 1 lists the results. In the second column we show the differences between our TEC, found by keeping the same biases calculated for November 20, and the TEC found using the GIM technique created independently for the quiet night of November 24-25. We see that the biases remain almost identical. In two of the five satellites we see differences of less than 1 TECU, with the other three within 2 TEC units. It seems that we can use the biases calculated on a quiet day fairly confidently for several days.

Table 1. TEC Differences for a Quiet Night (November 24-25) and an Active Night (November 22-23)

\begin{tabular}{lcc}
\hline PRN & Quiet & Active \\
\hline 2 & 1.2 & 0.7 \\
3 & $\ldots$ & 0.3 \\
19 & 1.5 & 4.1 \\
22 & 2.0 & 1.1 \\
27 & 0.8 & 0.4 \\
31 & 0.8 & 2.9 \\
Average & 1.3 & 1.6 \\
\hline
\end{tabular}

aThe difference shown is between values obtained by propagating the biases obtained on November 20 and an independent application of the GIM technique for the individual day. PRN refers to the pseudorandom number that is given to each GPS satellite. 
The results from the storm night, shown in column 3 , provide interesting insights into possible limitations of modeling during ionospheric disturbances. The largest errors occurred in space vehicles (SVs) 19 and 31 . These are the two satellites for which the line-of-sight TEC was most disturbed. Several cycle slips are also present in the data for these two satellites. Consequently, some of the differences can possibly be attributed to incorrect handling of these slips. For the remaining four satellites, comparing biases calculated independently for this day and using the biases from November 20, we see that the values are fairly close. These four satellites have differences well within the tolerance of the method. Even for SVs 2 and 27, for which the line-of-sight TEC shows great fluctuations and large gradients, the differences are quite small ( 0.7 and 0.4 TECU, respectively).

It is interesting to note that the average error is $30 \%$ higher on the active day, even though it occurred closer in time to the calibration date. Coco et al. [1991] suggest that the bias error contains not only the receiver and satellite errors but also an error due to mismodeling of the ionosphere. It is possible that larger errors occur in satellites whose lines-of-sight experience greater fluctuations in the TEC because of these modeling effects.

Using the method described above with the GIM technique, we have determined the absolute TEC for
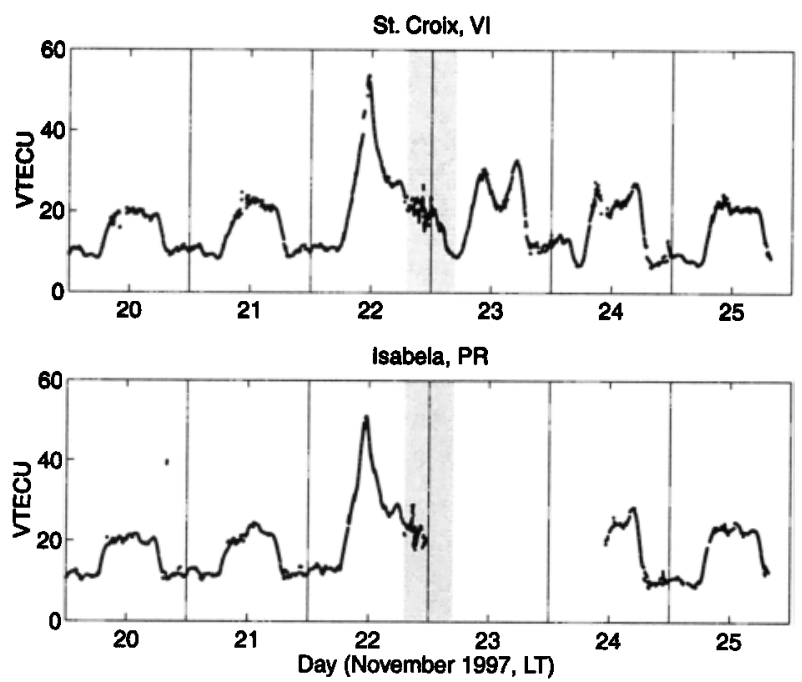

Figure 2. TEC profile for November 20-25, 1997. The highlighted region is the night of November 22-23. The vertical lines indicate local midnight. The Isabela receiver was down from the morning of November 23 until midday November 24. UT is 4 hours later than the local time plotted here. VI, Virgin Islands; PR, Puerto Rico.

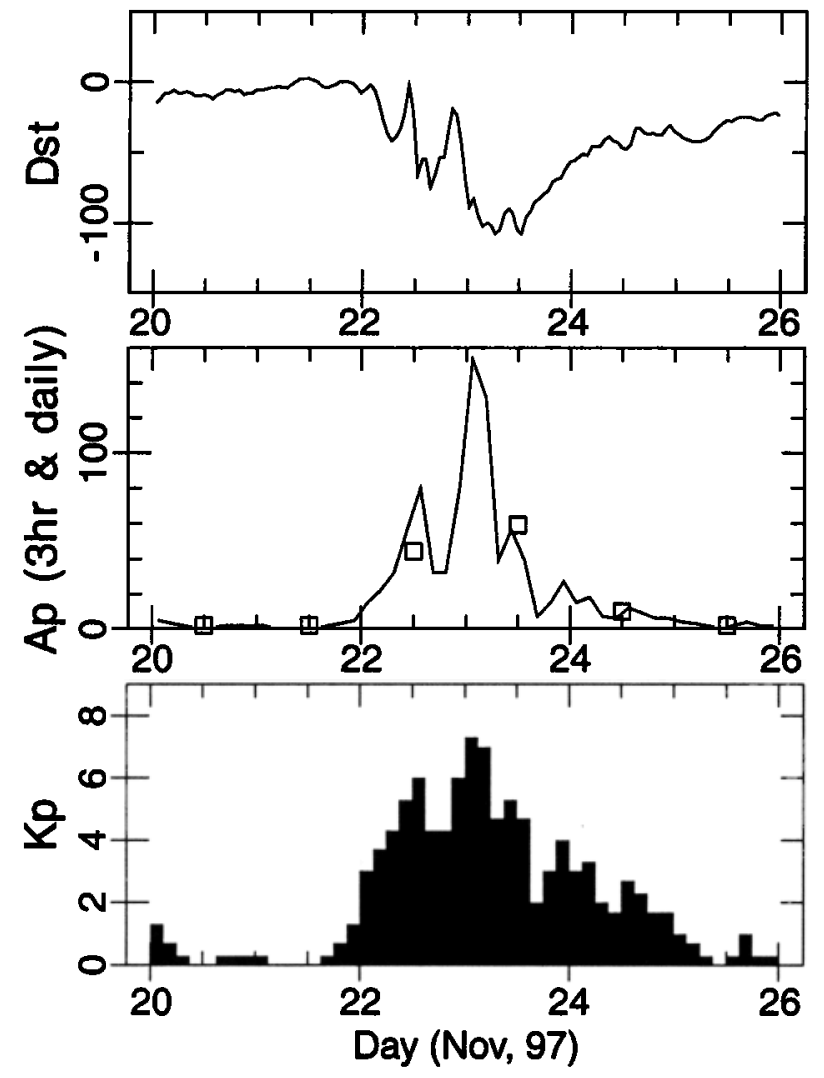

Figure 3. Geomagnetic indices: (top) $D s t$, (middle) $A p$, and (bottom) $K p$ for November 20-25, 1997.

all the satellites in view above an elevation angle of $25^{\circ}$ for a 6 day period. These results have been averaged and converted to vertical TEC, which is plotted in Figure 2 for both St. Croix, Virgin Islands and Isabela, Puerto Rico. By and large, when averaged like this, the two data sets are quite similar, further evidence that the method is working. The largest differences occur in the active nighttime period on November 22-23. This large difference between the TEC calculated for the two sites is to be expected on the basis of the huge differences in TEC seen for a common penetration point viewed from these nearby stations, as shown in Figure 1.

As seen in Figure 3, magnetic activity began at 0000 UT on November 22. The Dst (top panel) shows a storm beginning on November 22 at 0000 UT and reaching its maximum negative $D s t$ of -100 gamma on November 23 as late as 1200 UT. Over this same period the $A p$ and $K p$ indices show that this period was very disturbed. The $K p$ reaches 6 at 0000 

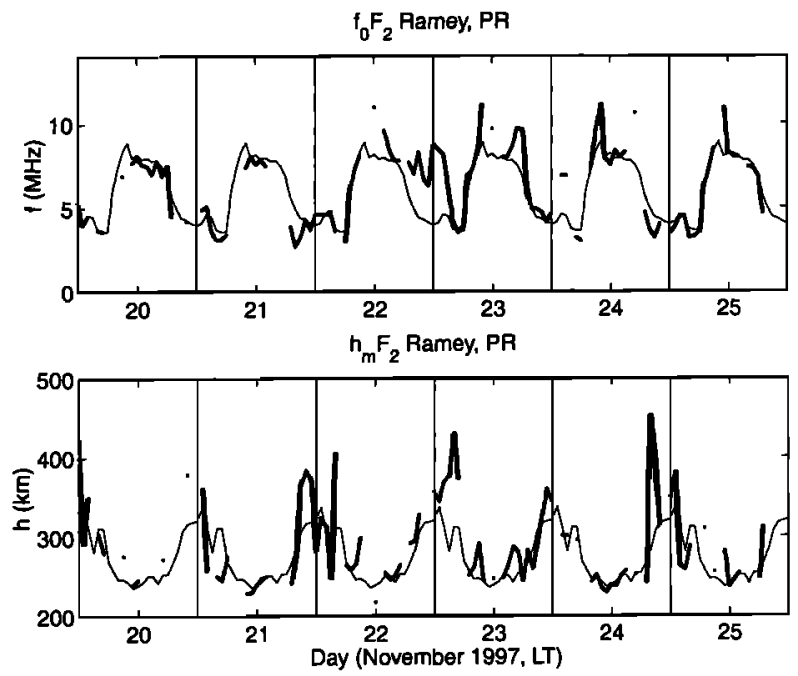

Figure 4. $f_{o} F_{2}$ and $h_{m} F_{2}$ plots for the 6 day period surrounding the event as observed at the Ramey Solar Observatory in Puerto Rico. The monthly average value is also plotted (repetitive curve). The vertical lines are local midnight.

UT on November 23. By November 25 the storm has diminished and, in fact, can be viewed as having recovered to its prestorm conditions. There was essentially no reaction to the storm's sudden commencement in the TEC or in $f_{o} F_{2}$ as measured by an ionosonde at the nearby Ramey Solar Observatory $\left(18.5^{\circ} \mathrm{N}, 292.8^{\circ} \mathrm{E}\right)$. This can be seen in Figure 4 , in which both $f_{o} F_{2}$ and $h_{m} F_{2}$ are plotted for that site for November 20-25. However, at the storm onset, near $0000 \mathrm{UT}$, the Caribbean ionosphere did surge to heights more than $100 \mathrm{~km}$ above the average height for the month (the repetitive curve). By early morning of the next day the TEC over the Caribbean sites was twice its average value. This huge peak occurs at the same local time (1200 LT) as the first of two well-defined peaks on the next day and less defined but perceptible double peaks on the next 2 days. These structures are called diurnal double maxima (DDM) in the literature [e.g., $P i$ et al., 1993] and are thought to be due to the effect of magnetic activity on the midlatitude ionosphere. These data certainly support this notion.

The premidnight values of $h_{m} F_{2}$ were elevated over the average on the night prior to the storm commencement. The near-dawn uplift on November 22 to over $400 \mathrm{~km}$ coincides with $100 \mathrm{nT}$ and $20 \mathrm{nT}$ magnetic field pulses in Iceland and at geostationary orbit, respectively (see Garcia et al. [2000] for more information on the event). This may be due to an eastward electric field penetrating the nightside, as is often observed [Kelley et al., 1979; Fejer, 1991]. Near dawn on November 22 , the $h_{m} F_{2}$ parameter exhibited a $50 \mathrm{~km}$ rise above the monthly average. Unfortunately, a data gap occurred at this time. The TEC data in Figure 2 show a doubling of the TEC over normal values around noon on November 22. This large increase in TEC is consistent with the Ramey data, which once data recording resumed, also show an increase in the $f_{o} F_{2}$ parameter shortly after noon. The TEC measured over Puerto Rico was not a local event. Indeed, a patch of intense ionization seems to move equatorward all the way from Greenland, leading up to the time of the large Caribbean peak [Garcia et al., 2000]. At sunset, neither the TEC nor $f_{o} F_{2}$ dropped as they usually do, further indicating the severity of the midlatitude disturbance. Figure 5 shows the tremendous variability of the TEC that night, which was similar to the variability of the 6300 airglow. An example is presented in Figure 6. Here the bright and dark airglow patches are correlated with high and low TEC values. It should be pointed out that the intensity of the 6300 airglow emission is also related to the height of the layer. However, in this case, the simultaneous TEC data show that the intensity variations are dominated by changes in electron content, rather than height changes. Because of the sharp gradients in the ionosphere the TEC differs
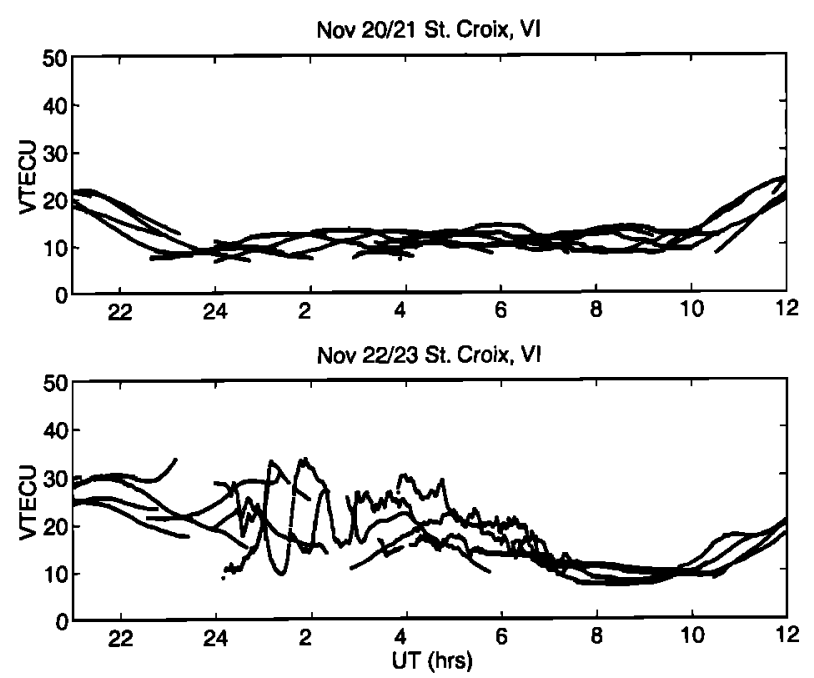

Figure 5. Vertical TEC calculated for all satellites in view from St. Croix, Virgin Islands over a 15 hour period for (top) a quiet night, November 20-21, 1997 and (bottom) the time period during intense TEC fluctuations on the night of November 22-23, 1997. 

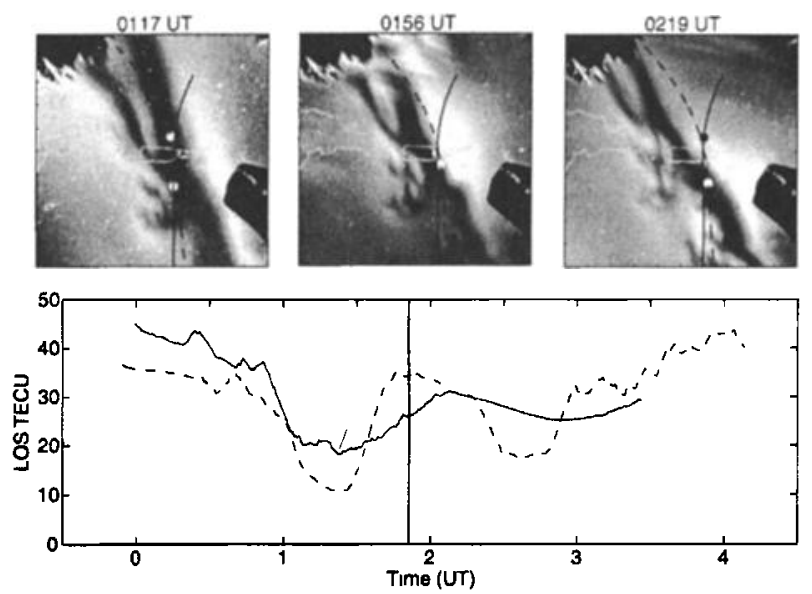

Figure 6. (top) Three airglow images with the trajectories of GPS satellites 15 (solid curves; viewed from Isabela, Puerto Rico) and 19 (dashed curves; viewed from St. Croix, Virgin Islands) superimposed. (bottom) Lineof-sight TEC for the satellite passes. The circles on the trajectories indicate the satellite positions at the times of the images. The vertical line corresponds to the time of closest approach between the two satellites.

by as much as 8 TEC units for the same ionosphere penetration point viewed from different locations.

\section{CIC Caribbean November 1997 GPS TEC Data-Model Comparison}

Before any data stream can be assimilated, it is critical that a data quality evaluation be made. We have undertaken initial steps to evaluate the quality of the calibrated GPS TEC data from the St. Croix site during this time period. All data used were slant path data. As already noted, the 2 earlier days were from a very quiet period prior to a major storm beginning on November 22 . Figure 3 highlights this temporal geomagnetic variability.

The task of quality controlling these data is complex. Ground truth data, such as those from the Arecibo incoherent scatter radar (ISR), do not necessarily follow the same slant paths and are not available for these nights, so these data cannot be used as a reference. In addition, at least 3 days (November 22,23 , and 24) are contaminated by active geophysical conditions. On the quiet days, however, one would expect to see a degree of repeatability and, perhaps, agreement with the general trends of a climatological model, such as diurnal variation and local spatial gradients. For this study, we have used the international reference ionosphere (IRI) climatological model. Therefore we approached the quality assessment by looking for common trends between each of the quiet days and then, subsequently, looking for trend differences with the IRI empirical model for these quiet days. Using this technique is different but not necessarily better than using comparisons with direct measurements, such as an ISR, TOPEX, or Faraday rotation measurements. In fact, comparisons of this nature have been carried out in the past [e.g., Lanyi and Roth, 1988; Ho et al., 1997; Vladimer et al., 1997; Iijima et al., 1999; Makela et al., 2000].

A first step in carrying out these comparisons is the need to synthesize GPS station-to-satellite ray paths through the IRI model. These synthetic ray paths then permit the integration of electron densities along the slant paths to produce model TEC
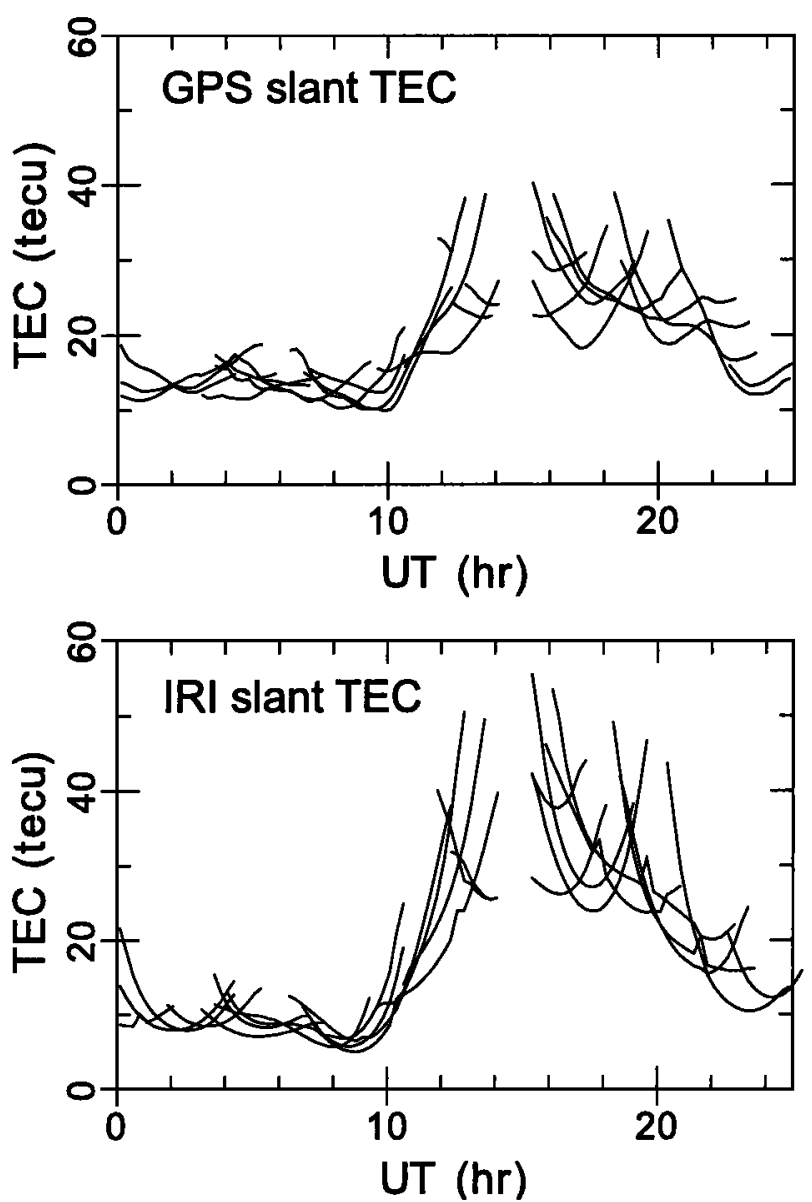

Figure 7. (top) Total electron content observed by the St. Croix GPS receiver on November 20, 1997, and (bottom) synthesized GPS TEC using the IRI model. 
values for the GPS slant paths. The software has been developed to handle arbitrary ground stations and realistic ray path elevation and azimuth angles. Figure 7 (upper panel) shows the observed St. Croix slant TEC for November 20. Each line segment corresponds to a continuous receiver observation period
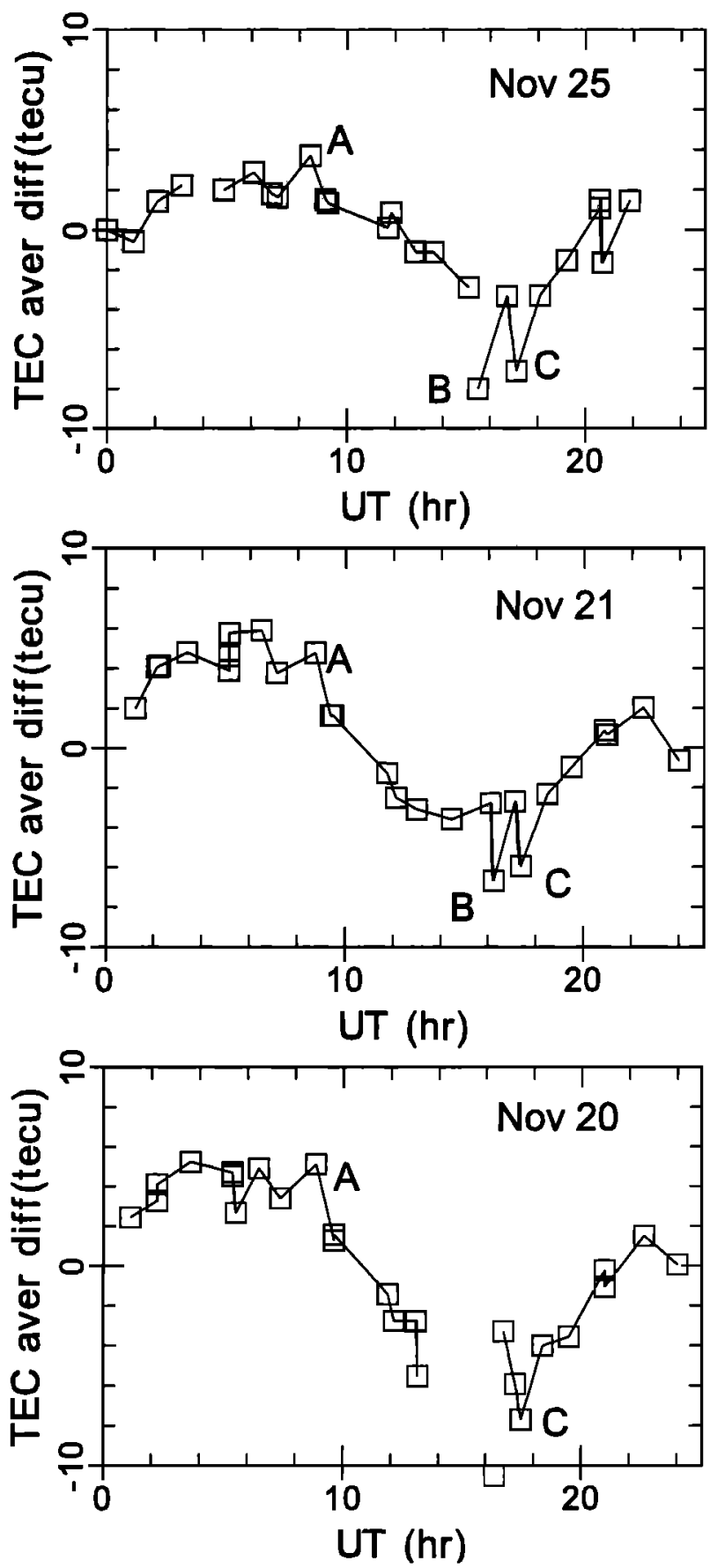

with a specific GPS satellite. That the line segments do not provide a unique diurnal curve is understandable because these different segments correspond to different elevation and azimuth angles. The slant TEC depends on the electron density along the ray path to the satellite [Lanyi and Roth, 1988]. Therefore the TEC has a strong dependence on the elevation angle to the satellite. The lower panel in Figure 7 shows the corresponding IRI slant TEC values. Note that IRI can only be integrated up to $1000 \mathrm{~km}$, whereas GPS satellites are located at radial distances of $20,200 \mathrm{~km}$. An inspection of Figure 7 reveals that a data gap exists between 1400 and $1500 \mathrm{UT}$ and that the nighttime TEC values occur between 0100 and $1000 \mathrm{UT}$, while daytime high TEC values extend from 1100 to 2200 UT. The IRI nighttime TEC values are lower than the measurements by a few TEC units, while the dayside IRI values exceed the observed GPS values.

As discussed in section 2, the analysis of GPS $L 1$ $L 2$ data to produce TEC yields highly accurate relative data, with the possibility of poorly defined offsets due to satellite and receiver biases. Therefore a logical analysis of Figure 7 data would be to difference the GPS and IRI TECs, rather than looking for scaling factors. If, in fact, offsets exist, they would probably be constant for all of the $30 \mathrm{~s}$ samples for one specific satellite. In Figure 8 these satellite offsets are shown for the average difference of GPS TEC minus IRI TEC for each satellite and plotted at the average UT of the satellite pass. Figure 8 shows these offsets for the 3 quiet days: November 20, 21, and 25. If the IRI and GPS agreed perfectly, the squares would form a line at 0 TEC difference. They do not; however, they do show a reasonably repeatable diurnal pattern. On all 3 days the GPS TEC exceed the IRI TEC values between 0000 and 1000 UT. Then, from 1000 to 2000 UT the IRI TEC exceed the observed values. This pattern could almost be viewed as a diurnal sine wave of amplitude 2.5 TEC units. Although most of the satellites lie along a smooth diurnal trend, at least three satellites are systematically disjoint from this curve. These have

Figure 8. Average satellite TEC difference between the observed GPS and IRI-modeled GPS for (top) November 25, (middle) November 21, and (bottom) November 20. Each square represents a different GPS satellite and includes data from $25^{\circ}-90^{\circ}$ elevation. 


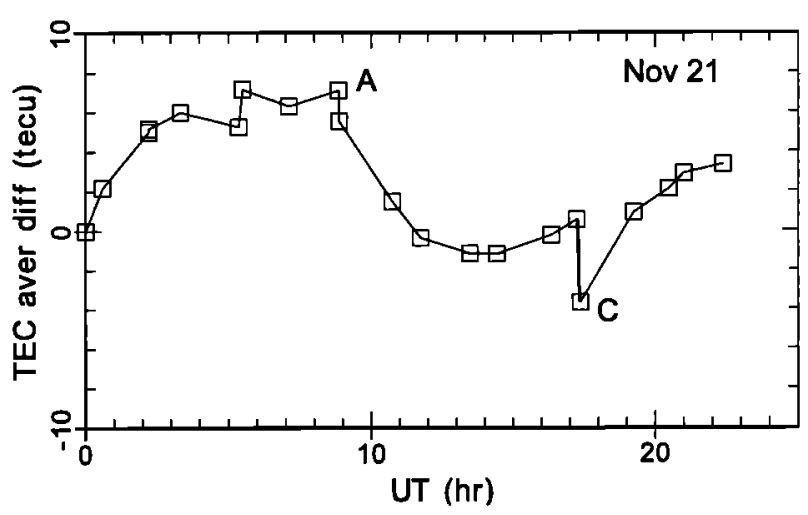

Figure 9. The average satellite TEC difference between the observed GPS and IRI-modeled GPS for November 21 for elevation angles from $25^{\circ}-90^{\circ}$ only.

been labeled as satellites A, B, and C in Figure 8 . Other satellites may deviate from a smooth curve on 1 or 2 , but not all, of the 3 days. With more quiet day data sets from other stations it should be possible to determine if satellites $\mathrm{A}, \mathrm{B}$, and $\mathrm{C}$ have been incorrectly calibrated with respect to the other satellites or if some other factor is responsible for their deviations. This would be a crucial step, prior to any assimilation work carried out on these data.

In Figure 8 all elevation angles between $25^{\circ}$ and $90^{\circ}$ were used. It may be argued that the very low elevation angle leads to very large slant TEC values and therefore the small differences in Figure 8 would not be meaningful. To compensate for this, the Figure 8 analysis was repeated using only data from elevation angles greater than $50^{\circ}$. Some of the satellites were then excluded from the analysis because the satellites never reached above $50^{\circ}$ as viewed from the ground station. Figure 9 shows the results for November 21 using this restricted elevation criterion. Satellites A and C still appear, but now satellite B is no longer present. For both of these satellites, the trend is the same as that found in the Figure 8 case. In Figure 9 the entire diurnal curve appears to be shifted up by 2.5 units. Indeed, the diurnal curve also looks considerably smoother.

Figures 8 and 9 demonstrate a means for quality control in the GPS TEC analysis. Even if the IRI is not exactly representative of the ionosphere, one would expect that, in general, the different satellites should reproduce this ionosphere in a systematic way. The repeated satellite $(A, B, C)$ departures from this systematic curve would therefore indicate a calibra- tion error. Basically, the quiet time ionosphere is well characterized, and the roughness exhibited by satellites $A, B$, and $C$ indicates that their offset bias values need correction. Otherwise, ingesting these data into an assimilation model would generate noise, creating an erroneous output from the model. The question of what the diurnal difference curve reveals is also significant. In both Figures 8 and 9 the modulation amplitude is \pm 2.5 TEC units. The Figure 7 observations show that the nighttime TECs are on the order of 11 TEC units, whereas the daytime values extend to 20 TEC units. A diurnal modulation of 5 TEC units, due to calibrations, would be rather large. Is this diurnal offset due to the plasmasphere? In Figure 9 the daytime offset appears to be close to zero. However, at night the GPS TEC exceeds that of the IRI. This might suggest a difference due to the plasmasphere. In fact, the magnitude of the difference (25 TECU) is consistent with that found by Lunt et al. [1999]. Unfortunately, this argument is false because the plasmasphere is a reasonably uniform torus encircling the equator, which would provide equal day
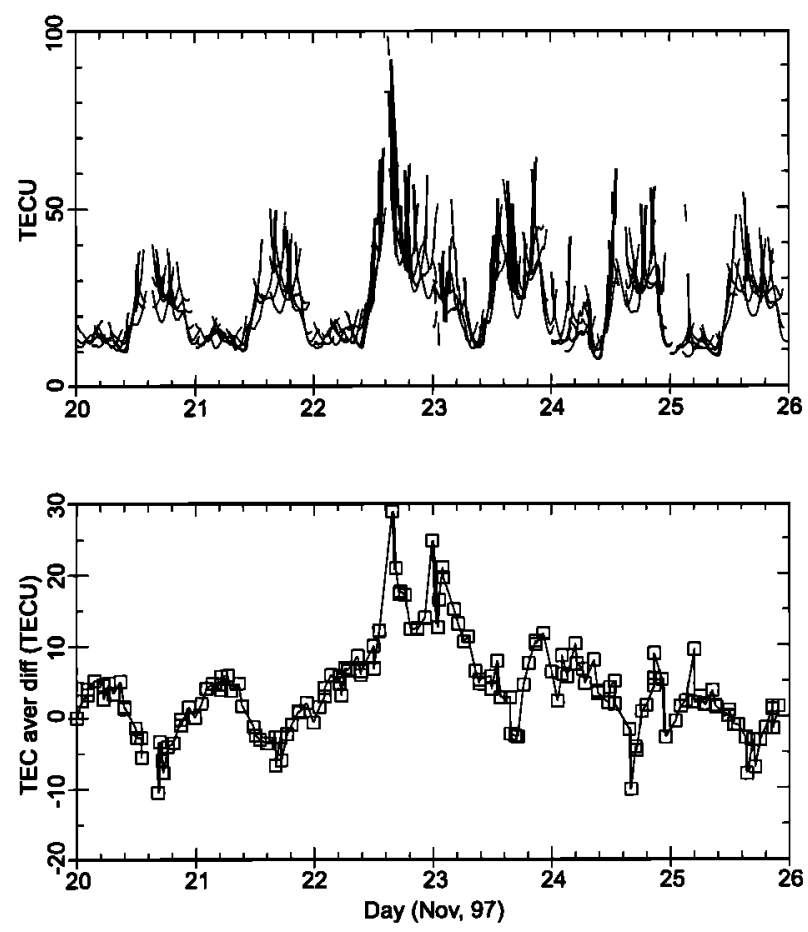

Figure 10. (top) GPS TEC for the entire study period of November 20-25, 1997, and (bottom) average satellite TEC difference between the observed and IRI-modeled TEC. 
and night TEC corrections. Significantly more work is needed to resolve this problem correctly.

Figure 10 shows the entire GPS TEC database for the study period (top panel) with the corresponding difference to IRI (lower panel). The diurnal patterns shown in Figure 8 for the 3 quiet days are evident in Figure 10, but on the disturbed days this is no longer true. During this disturbance the storm days clearly generate an ionosphere that is significantly greater (10-20 TEC units) than the IRI. To properly model these storm days, the neutral temperatures, densities, and winds as well as the plasma temperatures must be properly adjusted to obtain slant path TEC values from the ionospheric model that are consistent with the measurements. This work is in progress, and the GPS TEC data are, in fact, the basis for the assimilation being developed as Assimilation Ionosphere Model (AIM) 1.04L by the Space Environment Corporation as part of the AIM project, and as Global Assimilative Ionosphere Model (GAIM) by a team of scientists at the University of Southern California and the Jet Propulsion Laboratory as part of the GAIM project.

\section{Summary}

In this paper, we use GPS satellites to document remarkable variations of the total electron content in the ionosphere-plasmasphere during a period of high magnetic activity. The noontime TEC was double that at noon the previous day. By midafternoon, the activity had decreased, but it rebounded again near sunset. That night, the average TEC remained very high, at a level more typical of daytime than of nighttime values.

From a technical standpoint we show that it is quite feasible to calibrate satellite-receiver pairs using data from the GIM technique on a quiet day and to have the calibration hold to within 2 TEC units (RMS) for several days. This allows for reliable study of mesoscale structures during active periods without dependence on modeling during severe weather, which seems to be more difficult than quiet day modeling.

A fortuitous satellite-receiver pair combination allowed us to investigate a common ionosphere penetration point each day during the whole period. The agreement was quite good, except for the active day when the vertical TEC differed by 8 TEC units. The airglow data show that high TEC values corresponded to look angles through very bright airglow patches. In general, the bright (dark) airglow regions corresponded to high (low) TEC. This result is actually important since it implies that dynamical variations dominate the airglow patterns, not chemical changes.

In this study, we have not only seen how robust the GPS calibration procedures are during disturbed periods but also independently evaluated the quality of this calibration as a precursor to using such data in assimilation models. The results of this study identified at least three satellites in the database that appear to deviate significantly from the others. A diurnal modulation in the calibration appears systematically on each quiet day when referenced to the IRI slant path TECs. For the purpose of incorporating these data into models it must be determined whether the three apparently anomalous satellites are, in fact, anomalous. In addition, it must be determined whether the diurnal modulation is simply an artifact of comparing the data to IRI or a calibration issue. However, both of these effects are of lower order to the extensive storm information obtained by GPS slant TEC from only two ground stations.

Other remarkable aspects of these data are the enormous range of TEC values registered by a receiver looking at many satellites and the differences registered by two nearby receivers looking at the same ionospheric penetration point. The simultaneous airglow data reveal unexpected and collocated airglow variations of great complexity.

Acknowledgments. We thank Craig Teply, Steve Collins, and Jonathan Friedman for helping with the airglow imager throughout its stay at the Arecibo Observatory. Research at Cornell University was supported under ONR grant N00014-92-J-1822 and AFOSR grant 97NM219. J.J.M. is supported under a National Science Foundation Graduate Research Fellowship. Work at the Space Environment Corporation was supported by ONR contract N00014-98-C-0085. Research conducted at the Jet Propulsion Laboratory is under a contract with the National Aeronautics and Space Administration.

\section{References}

Coco, D. S., C. Coker, S. R. Dahlke, and J. R. Clynch, Variablity of GPS satellite differential group delay biases, IEEE Transactions on Aerospace and Electronic Systems, 27(6), 931-938, 1991.

Fejer, B. G., Low latitude electrodynamic plasma drifts: A review, J. Atmos. Terr. Phys., 53(8), 677-693, 1991. Garcia, F. J., M. C. Kelley, J. J. Makela, P. J. Sul- 
$\tan$, X. Pi, and S. Musman, Mesoscale structure of the midlatitude ionosphere during high geomagnetic activity: Airglow and GPS observations, J. Geophys. Res., 105 (A8), 2000.

Ho, C. M., A. J. Mannucci, U. J. Lindqwister, X. Pi, and B. J. Tsurutan, Global ionosphere perturbations monitored by the worldwide GPS network, Geophys. Res. Lett., 23(22), 3219-3122, 1996.

Ho, C. M., B. D. Wilson, A. J. Mannucci, U. J. Lindqwister, and D. N. Yuan, A comparative study of ionospheric total electron content measurements using global ionospheric maps of GPS and TOPEX radar, and the Bent model, Radio Sci., 32(4), 1499-1512, 1997.

Iijima, B. A., I. L. Harris, C. M. Ho, U. J. Lindqwister, A. J. Mannucci, X. Pi, M. Reyes, L. C. Sparks, and B. D. Wilson, Automated daily process for global ionospheric total electron content maps and satellite ocean altimeter ionospheric calibration based on Global Positioning System data, J. Atmos. Solar-Terr. Phys., 61 (16), 1205-1218, 1999.

Kelley, M. C., B. G. Fejer, and C. A. Gonzales, An explanation for anomalous equatorial jonospheric electric fields associated with a northward turning of the interplanetray magnetic field, Geophys. Res. Lett., 6(4), 301-304, 1979.

Kelley, M. C., F. Garcia, J. J. Makela, T. Fan, E. Mak, C. Sia, and D. Alcocer, Highly structured tropical airglow and TEC signatures during strong geomagnetic activity, Geophys. Res. Lett., 27(4), 465-468, 2000.

Lanyi, G. E., and T. Roth, A comparison of mapped and measured total ionospheric electron content using global positioning system and beacon satellite observations, Radio Sci., 23(4), 483-492, 1988.

Lunt, N., L. Kersley, G. J. Bishop, and A. J. Mazzella, The contribution of the protonosphere to GPS total electron content: Experimental measurements, Radio Sci., 34(5), 1273-1280, 1999.

Makela, J. J., S. A. González, B. MacPherson, X. Pi, M. C. Kelley, and P. J. Sultan, Intercomparisons of total electron content measurements using the Arecibo incoherent scatter radar and GPS, Geophys. Res. Lett., 27(18), 2841-5844, 2000.

Mannucci, A. J., B. D. Wilson, D. N. Yuan, C. H. Ho, U. J. Lindqwister, and T. F. Runge, A global mapping technique for GPS-derived ionospheric electron content measurements, Radio Sci., 33(3), 565-582, 1998.

Pi, X., M. Mendillo, M. W. Fox, and D. N. Anderson, Diurnal double maxima patterns in the $\mathrm{F}$ region ionosphere: Substorm-related aspects, J. Geophys. Res., 98(A8), 13,677-13,691, 1993.

Sardon, E., and N. Zarraoa, Estimation of total electron content using GPS data: How stable are the differential satellite and receiver instrumental biases?, Radio Sci., 32(5), 1899-1910, 1997.

Vladimer, J. A., M. C. Lee, P. H. Doherty, D. T. Decker, and D. N. Anderson, Comparisons of TOPEX and Global Positioning System total electron content measurements at equatorial anomaly latitudes, Radio Sci., 32(6), 2209-2220, 1997.

Wilson, B. D., A. J. Mannucci, and C. D. Edwards, Subdaily northern hemisphere ionospheric maps using an extensive network of GPS receivers, Radio Sci., 30(3), 639-648, 1995.

M. C. Kelley and J. J. Makela, School of Electrical and Computer Engineering, Cornell University, 318 Rhodes Hall, Ithaca, NY 14853-3801. (mikek@ece.cornell.edu; makela@ece.cornell.edu)

A. J. Mannucci and X. Pi, Jet Propulsion Laboratory, M/S 138-308, 4800 Oak Grove Drive, Pasadena, CA 91109-8099. (tony.mannucci@jpl.nasa.gov; xiaoqing.pi@jpl.nasa.gov)

J. J. Sojka, Space Environment Corporation, 399 North Main, Suite 325, Logan, UT 84321. (jan@spacenv.com)

(Received December 22, 1999; revised September 29, 2000; accepted October 16, 2000.) 